\title{
Dihydromyricetin modulates p62 and autophagy crosstalk with the Keap-1/Nrf2 pathway to alleviate ethanol-induced hepatic injury
}

Ping Qiu ${ }^{\mathrm{a}, 1}$,Yu Dong ${ }^{\mathrm{b}, 1}$, Bo Li ${ }^{\mathrm{a}}$, Xian-jie Kang ${ }^{\mathrm{a}}$, Chao Gu ${ }^{\mathrm{a}}$, Tao Zhu ${ }^{\mathrm{a}}$, Yun-yun Luo ${ }^{\mathrm{a}}$, Min-xia Pang $^{\mathrm{a}}$, Wei-feng $\mathrm{Du}^{\mathrm{a}}$, Wei-hong $\mathrm{Ge}^{\mathrm{a}}$,

${ }^{\mathrm{a}}$ Zhejiang Chinese Medical University, Hangzhou, Zhejiang Province 310053, China

b Department of Medicine, Zhejiang Academy of Traditional Chinese Medicine, Hangzhou, Zhejiang Province 310007, China

* Corresponding author at: Zhejiang Chinese Medical University, Hangzhou, Zhejiang Province 310053, China.

E-mail addresses: geweihong@ hotmail.com (W.-H. Ge).

${ }^{1}$ These authors contributed equally to this work.

Keywords: Dihydromyricetin; Liver injury; p62; Keap-1/Nrf2 pathway; Autophagy

Abbreviations: ALD, alcoholic liver disease; ALP, alkaline phosphatase; ALT, alanine aminotransferase; ARE, antioxidant response element; AST, aspartate aminotransferase; CYP2E1, cytochrome P450, family 2, subfamily E, polypeptide 1; DAB, 3, 3'-diaminobenzidine; DAPI, 4', 6-diamidino-2-phenylindole, dihydrochloride; DMY, dihydromyricetin; DTT, dithiothreitol; ECL, enhanced chemiluminescence; EDTA, ethylene diamine tetraacetic acid; EGTA, ethylene glycol-bis(2-aminoethyl ether)-N, N, N', N'-tetraacetic acid; ELISA, enzyme-linked immunosorbent assay; GSH, glutathiose; H\&E, haematoxylin-eosin; HEPES, 4-(2-hydroxyethyl)-1-piperazineethanesulfonic acid; HO-1, haeme oxygenase 1; HPLC, high performance liquid chromatography; IgG, immunoglobulin G; IL-1 $\beta$, interleukin 1 beta; IL-6, interleukin 6; Kelch-Like Ech-Associated Protein 1, Keap-1; LC3B, light chain 3-beta; LDH, lactate dehydrogenase; LPS, lipopolysaccharide; MDA, malonydialdehyde; NADPH, nicotinamide adenine dinucleotide 2"-phosphate reduced tetrasodium salt; NF- $\mathrm{KB}$, nuclear factor- kappabinding; Nrf2, erythroid 2-related factor 2; OCT, opti-mum cutting temperature; PBS, phosphate buffer saline; ROS, reactive oxygen species; RIPA, radio-immunoprecipitation assay; TBARS, thiobarbituric acid reactive substances; TEM, transmission electron microscope. 


\begin{abstract}
Increasing evidence has demonstrated that dihydromyricetin (DMY) contains highly effective antioxidative, anti-inflammatory, anti-microbial and anti-diabetic properties. Nevertheless, the underlying hepatoprotective mechanisms of DMY have infrequently been reported thus far. In the present study, C57BL/6 mice were fed with the Lieber-DeCarli diet containing alcohol or isocaloric maltose dextrin as a control diet with or without DMY (75 and $150 \mathrm{mg} / \mathrm{kg} / \mathrm{d}$ bw) for 6 weeks. DMY significantly attenuated hepatic enzyme release, hepatic lipid peroxidation and triglyceride deposition induced by chronic alcohol exposure. In addition, DMY dramatically attenuated the alcohol-triggered elevation of the level of inflammatory cytokines and partially recovered hepatic pathological changes. Notably, DMY remarkably modified aberrant expression of CYP2E1, Keap-1 and HO-1 in the liver and simultaneously ameliorated disordered nuclear localization of NF-kB and Nrf2 to exert its hepatoprotective effects. Further mechanistic exploration suggested that DMY activated Nrf2, possibly mediated through the autophagy pathway. Analysis of the crosstalk among p62, Keap-1 and Nrf2 demonstrated that the p62 upregulation caused by DMY contributes to a positive feedback loop in Nrf2 activation. In summary, DMY likely modulates p62 and autophagy crosstalk with the Keap-1/Nrf2 pathway to alleviate liver steatosis and the inflammatory response in the pathological progression of ALD.
\end{abstract}




\section{Introduction}

Alcoholic liver disease (ALD), which is caused by excessive ethanol consumption, constitutes a major public health problem and is responsible for high morbidity and mortality worldwide (Orman et al., 2013). Hepatic steatosis as the initial pathological phase of ALD (more than 90\%), which is characterized by an excessive build-up of lipid droplets in hepatocytes, and $35 \%$ of this population progresses to steatohepatitis, fibrosis, cirrhosis and possibly even hepatocellular carcinoma (HCC) (Gao and Bataller, 2012). In the past decade, the pathogenesis of ALD has emphasized oxidative stress, the inflammatory response, gut microbiota and the LPS pathway, and the innate immune system (Dhanda et al., 2012). In particular, oxidative metabolites largely triggered by CYP2E1, such as toxic aldehydes and reactive oxygen species (ROS), are intimately involved in disruption of multiple intracellular signalling pathways. (Ceni et al., 2014).

Notably, recent evidence has demonstrated that activation of nuclear erythroid 2-related factor 2 (Nrf2) is crucial in diminishing the oxidative stress response during the catalytic cycle of CYP2E1 (Bataille and Manautou, 2012; Kobayash and Yamamoto, 2005). Briefly, Nrf2 has currently emerged as a critical therapeutic regulator for treating ALD, with a series of downstream targets aimed at cytoprotection, namely haeme oxygenase 1 (HO-1) and GSH. In addition, numerous lines of evidence have highlighted that chronic consumption of alcohol results in down-regulation of Nrf2 and its targets (Bardag-Gorce et al., 2011). Intriguingly, Nrf2 activators, such as sulforaphane, are effective in alleviating chronic liver damage by stimulating Nrf2 nuclear translocation and subsequently protect against CYP2E1-dependent binge alcohol-induced liver steatosis (Zhou et al., 2014). Furthermore, nuclear transcription factor- $\mathrm{kB}$ (NF- $\kappa \mathrm{B}$ ) is recognized as an essential transcriptional regulator involved in oxidative stress, and NF- $\mathrm{KB}$ activation is followed by the release of a variety of proinflammatory cytokines, such as TNF- $\alpha$, IL-6, and IL-1 $\beta$ (Gyamfi and Wan, 2010; Li, et al., 2015).

In particular, recent studies have revealed that autophagy plays a cytoprotective role against ethanol-induced liver damage via removal of damaged mitochondria and lipid droplets (Nepal \& Park, 2013). Furthermore, several lines of evidence have suggested that the Nrf2 signalling pathway can also be activated by the p62-mediated pathway because p62 competes with Nrf2 for Kelch-Like Ech-Associated Protein 1 (Keap-1) binding under oxidative stress conditions (Jain et al., 2010). More importantly, Keap-1 interacts with both LC3B and p62, leading to autophagic degradation of endogenous Keap-1, which can create a positive feedback loop of Nrf2 activation (Fan et al., 2010, Hayashi et al., 2015). Recently, modulation of the crosstalk among p62, Keap-1, and Nrf2 has been shown to be a new therapeutic target. Intriguingly, several drugs have been identified that exert protective properties that are likely mediated through the p62/Keap1/Nrf2 pathway, such as Fenofibrate and Ezetimibe (Park et al., 2015, Lee et al., 2016). In general, the p62/Keap-1 axis likely plays an essential role in protecting cells against oxidative stress damage. 
Remarkably, an extremely novel therapeutic option for preventing liver damage could lie in natural plant components, which are considered to possess multi-targeted activity and low toxicity, such as tanshinone and lucidone (Wang et al., 2016; Kumar et al., 2012). Intriguingly, dihydromyricetin (DMY) is the most abundant bioactive flavonoid in A. grossedentata. It has been reported that DMY is highly effective in multiple protective activities, including relieving alcoholism and antioxidative, anti-inflammatory, anti-microbial and anti-diabetic activities (Shen et al., 2012; Jiang et al., 2014; Shi et al., 2015). Despite limited knowledge of the effects and mechanisms of DMY, it has emerged as a promising therapeutic agent for ethanol-induced hepatotoxicity. In our study, we utilized C57BL/6 mice fed the Lieber-DeCarli liquid diet to evaluate the hepatoprotective effect of DMY and to further explore the underlying molecular mechanisms. Then, we investigated whether the protection provided by DMY against ethanol-induced hepatic steatosis and inflammatory response is associated with modulation of p62 and autophagy crosstalk with the Keap-1/Nrf2 pathway.

\section{Materials and methods}

\subsection{Reagents}

DMY (purity>95\%) used for the animal experiments was purchased from Nanjing Zelang Medical Technology Co., Ltd. (Nanjing, China). All other chemicals were reagent grade.

\subsection{Chromatographic analysis of DMY}

The DMY content was analysed with an Agilent 1100 series HPLC system (DE40525750, Agilent, USA) and a Welch Materials XB C18 column $(250 \mathrm{~mm} \times 4.6 \mathrm{~mm}, 5 \mu \mathrm{m})$. The flow rate and column temperature were set at $1 \mathrm{ml} / \mathrm{min}$ at a temperature of $25{ }^{\circ} \mathrm{C}$. The solvent system was methanol/ $/ 0.1 \%$ phosphoric acid (23:73). HPLC-grade methanol was purchased from Merck (Germany). Water was purified with a Milli-Q water purification system (Millipore, USA). Analytical-grade aqueous phosphoric acid and ethanol were purchased from Guangfu Technology Co., Ltd. (Tianjin, China). In the HPLC chromatogram, a wavelength of $290 \mathrm{~nm}$ was monitored for quantitative analysis of DMY. The injection volume was $10 \mu \mathrm{L}$ for each sample solution.

\subsection{Animals and experimental design}

Forty-eight specific pathogen-free male C57BL/6 mice were purchased from Shanghai Si-Lai-Ke Experimental Animal Ltd. (Shanghai China). The mice were housed in temperature-, light-, and humidity-controlled conditions with a 12-h light/dark cycle. The mice were randomized into four groups and placed three per cage in every group after one week of acclimation. Mice in the control group had pair-fed with the isocaloric liquid control diet, while the ethanol group and 
DMY groups received the Lieber-DeCarli liquid alcohol diet, which contained increasing concentrations of ethanol ( $1 \%$ for $2 \mathrm{~d}, 2 \%$ for $2 \mathrm{~d}, 4 \%$ for $7 \mathrm{~d}$ and then $4 \%$ for an additional 6 weeks). The reagents in the Lieber-DeCarli formula were obtained from Trophic Animal Feed High-Tech Co., Ltd. (Nantong, China).

Mice in the DMY groups were fed two different doses $(75$ and $150 \mathrm{mg} / \mathrm{kg}$ BW per day, respectively) of DMY by incorporating it into the diet. Simultaneously, mice in the control groups received an equivalent amount of physiological saline. All experimental procedures were approved by the National Institutes of Health Guidelines for the Care and Use of Laboratory Animals.

At the end of the experimental period, the mice were sacrificed after fasting for $12 \mathrm{~h}$. The serum was prepared by centrifugation at $1000 \times \mathrm{g}$ at $4{ }^{\circ} \mathrm{C}$ for 15 min (Beckman, Germany) and was stored at $-80^{\circ} \mathrm{C}$ for biochemical detection. The livers were immediately dissected and then fixed in $10 \%$ neutral buffered formalin for histopathological examination. The remainder of the fresh liver tissues was snap-frozen in liquid nitrogen, followed by preservation at $-80^{\circ} \mathrm{C}$.

\subsection{Determination of serum biomarkers}

The activities of alanine aminotransferase (ALT), aspartate aminotransferase (AST), alkaline phosphatase (ALP) and lactate dehydrogenase (LDH) in the serum samples were measured with an automatic biochemistry analyser according to the instructions provided by commercial assay kits (Hitachi 7020, Tokyo, Japan)

\subsection{Lipid peroxidation levels and Oil Red O staining}

The liver samples were homogenized in $0.15 \mathrm{mM} \mathrm{NaCl}$ (liver weight: $\mathrm{NaCl}$ volume $=1: 9$ ) at a temperature of $4{ }^{\circ} \mathrm{C}$ and centrifuged at $2000 \times \mathrm{g}$ for $15 \mathrm{~min}$. The $10 \%(\mathrm{w} / \mathrm{v})$ liver homogenate was collected from the supernatant, and the protein concentration was detected with a $\mathrm{BCA}^{\mathrm{TM}}$ protein assay kit. The MDA content was detected through the measurement of thiobarbituric acid reactive substances (TBARS) and is described as $\mathrm{nmol} / \mathrm{mg}$ protein (Jiancheng, Nanjing, China). Meanwhile, the GSH level was measured using a commercial assay kit (Beyotime, Nanjing, China) and is expressed as $\mathrm{nmol} / \mathrm{mg}$ protein.

For Oil Red O staining, liver tissues were embedded in OCT compound and 8- $\mu \mathrm{m}$-thick cryosections were prepared and subsequently stained with Oil Red $\mathrm{O}$ for lipid content determination. (Sigma Chemical, Co.). Oil Red O evaluations of the liver sections were conducted by a registered pathologist. Afterwards, the Oil Red O-stained area was photographed with a microscope at 200x magnification and analysed with Image-Pro Plus software.

\subsection{Hepatic histopathological evaluation and examination of biomarkers}


Liver sections were fixed in $10 \%$ neutral buffered formalin solution for a minimum of $24 \mathrm{~h}$, embedded in paraffin wax, sectioned at 4- $\mu \mathrm{m}$ thickness, stained with haematoxylin-eosin (H\&E) and digitally photographed with a light microscopic at a total magnification of 400x.

The liver samples were homogenized in $1 \mathrm{~mL}$ of $1 \times$ PBS and centrifuged at $12,000 \times \mathrm{g}$ for 15 $\min$ at $4{ }^{\circ} \mathrm{C}$, and the supernatants were prepared for the assay. The levels of interleukin 1 beta (IL-1 $\beta$ ) and interleukin 6 (IL-6) in the livers were detected by a fluorescence reader using a mouse enzyme-linked immunosorbent assay (ELISA) kit (Yuanye, Shanghai, China) and are expressed as $\mathrm{pg} / \mathrm{ml}$ and $\mathrm{pg} / \mathrm{mg}$, respectively.

\subsection{Western blot analysis}

Small pieces of a frozen liver sample were rinsed in ice-cold $10 \mathrm{mM}$ Tris- $\mathrm{HCl}(\mathrm{pH}$ 7.4) and homogenized with RIPA lysis buffer containing fresh cocktail protein inhibitors. The mixture was centrifuged at $12,000 \times \mathrm{g}$ for $15 \mathrm{~min}$, and the supernatant was kept as the total protein extract at $-80{ }^{\circ} \mathrm{C}$. Nuclear protein extraction was performed as follows: the liver was homogenized in hypotonic lysis buffer $\left(1.5 \mathrm{mM} \mathrm{MgCl}_{2}, 10 \mathrm{mM} \mathrm{KCl}, 10 \mathrm{mM}\right.$ HEPES, $1 \mathrm{mM}$ dithiothreitol, and $1 \%$ cocktail protein protease inhibitor, $\mathrm{pH}$ 7.9) and centrifuged at 10,000×g for $20 \mathrm{~min}$. The resultant nuclear pellet was lysed and incubated in nuclear extraction buffer on ice $(1.5 \mathrm{mM} \mathrm{MgCl}, 20 \mathrm{mM}$ HEPES (pH 7.9), 0.1 mM EDTA, 0.1 mM EGTA, 0.5\% Nonidet P-40, 1 mM dithiothreitol (DTT) and $1 \%$ cocktail protein protease inhibitors, $\mathrm{pH}$ 7.9). The mixture was vortexed and centrifuged at $20,000 \times \mathrm{g}$ for $15 \mathrm{~min}$. The supernatant (nuclear fraction) was collected and stored at $-80{ }^{\circ} \mathrm{C}$ for western blot analysis. The protein concentrations were quantified using a Bradford assay. (Pierce, Rockford, USA).

The protein samples (approximately $50 \mu \mathrm{g}$ ) were then separated by electrophoresis in a $10 \%$ denatured polyacrylamide gel and then transferred onto a $0.22 \mu \mathrm{m}$ polyvinylidene fluoride membrane (Bio-Rad, Germany). The membranes were blocked with a 5\% skim milk solution for 1 $\mathrm{h}$ at room temperature. Subsequently, the membranes were incubated with the respective primary antibodies overnight at $4{ }^{\circ} \mathrm{C}$, including antibodies against p62, LC3B, Nrf2 (Abcam, MA, USA), NF-кB (Santa Cruz, CA, USA), Tubulin (Affinity, CO, USA), CYP2E1, Keap-1, HO-1, Beclin 1, $\beta$-actin and Lamin B (Bioworld, Jiangsu, China). After washing three times with $1 \times$ TBST, the membranes were incubated with secondary antibody (Bioworld, Jiangsu, China) at room temperature for $2 \mathrm{~h}$. The protein bands were visualized using enhanced chemiluminescence (ECL) detection reagents (Millipore, Billerica, USA), and the results are expressed as fold changes normalized to the expression of Tubulin, $\beta$-actin or Lamin B1.

\subsection{Total RNA Extraction and RT-PCR Analysis}

Total RNA was isolated from liver tissues using TRIzol® reagent and was reverse transcribed with a cDNA synthesis kit (Takara, Japan). Subsequently, the resulting cDNA was subjected to 
qPCR analysis using SYBR® Green and a CFX96TM real-time PCR system (Bio-Rad, CA, USA). The sequences of the primers used for mouse samples were as follows: Keap-1, 5'-CAACTTCGCGGAGCAGATCG-3' and 5'-AGCTGGCAGTGTGACAGGTT-3'; and GAPDH, 5'-GGAAAGCTGTGGCGTGATGG-3' and 5'-AGCTCTGGGATGACCTTGCC-3'. Additionally, the relative expression levels of keap-1 were calculated with the $2^{-\Delta \Delta}{ }^{\mathrm{CT}}$ method and then normalized to the expression of GAPDH.

\subsection{Immunohistochemistry analysis of Keap-1 in the liver}

The liver slices were deparaffinised, rehydrated and subsequently subjected to $0.01 \mathrm{~mol} / \mathrm{l}$ citrate $(\mathrm{pH}=6.0)$ in a microwave oven for $20 \mathrm{~min}$ for antigen retrieval. Briefly, slices were immersed in 3\% hydrogen peroxide $\left(\mathrm{H}_{2} \mathrm{O}_{2}\right)$ for $10 \mathrm{~min}$ to block endogenous peroxidase activity at room temperature, and normal goat serum was added to block non-specific protein binding. Afterwards, the slices were incubated with anti-Keap-1 antibody (Bioworld, Jiangsu, China) at $4{ }^{\circ} \mathrm{C}$ overnight. After rinsing with PBS, the slices were incubated with secondary antibody (Invitrogen, CA, USA) at $37{ }^{\circ} \mathrm{C}$ for $1 \mathrm{~h}$ and subsequently stained with DAB (Zhongshan Golden Bridge, Beijing, China) for $10 \mathrm{~min}$. Before dehydration, the frozen tissue sections were counterstained with haematoxylin, and then, photographs were taken using a high-power light microscope at random fields to further investigate the localization of Keap-1. Morphometric analysis was performed using semi-quantitative Image-ProPlus software.

\subsection{Examination of the liver using transmission electron microscopy (TEM)}

Liver tissues sections $(1 \times 1 \times 1 \mathrm{~mm})$ were cut for ultrastructural examination. Subsequently, they were fixed in $2.5 \%$ glutaraldehyde ( $0.1 \mathrm{M}$ sodium cacodylate buffer, $\mathrm{pH} 7.4)$ and post-fixed in $1 \%$ osmium tetraoxide ( $0.1 \mathrm{M}$ sodium cacodylate buffer, $\mathrm{pH}$ 7.4). The liver samples were hydrated with a graded series of alcohol and acetone solutions and embedded in araldite. Ultrathin sections cut with an ultramicrotome (Leica UCT, Germany) were stained with uranyl acetate and lead citrate and examined and photographed with a Hitachi 7600 transmission electron microscope (Hitachi High-Technologies Corp., Tokyo, Japan).

\subsection{Immunofluorescence analysis of NF- $\kappa B$ and Nrf2 in the liver}

For immunofluorescent labelling, cryostat sections of the liver were cut into $10-\mu \mathrm{m}$-thick sections and then incubated with primary rabbit Nrf2 and NF- $\mathrm{kB}$ antibodies at $4{ }^{\circ} \mathrm{C}$ overnight. Afterwards, cryostat sections were incubated with secondary antibodies, Alexa Fluor ${ }^{\circledR} 488$-conjugated goat anti-rabbit $\operatorname{IgG}$ (Abbkine, Wuhan, China) and goat anti-mouse IgG-FITC (Abcam, MA, USA), respectively. Then, the slices were incubated with DAPI (Sigma, 
MO, USA) for $8 \mathrm{~min}$ at room temperature and extensively rinsed with $1 \times \mathrm{PBS}$. The experiment was performed following the manufacturer's protocol and was observed and digitally photographed with a laser scanning confocal microscope (LEICA, Mannheim, Germany) at a total magnification of $400 \times$.

\subsection{Statistical analysis}

All data were analysed with GraphPad Prism 5.0 software (GraphPad Software, San Diego, California, USA). All statistical comparisons were performed using one-way analysis of variance with a post hoc Dunnett's test. Values of $\mathrm{P}<0.05$ were considered statistically significant.

\section{Results}

\subsection{Preliminary characterization of DMY}

The DMY content was analysed by HPLC in this study, and the chemical analysis results determined that the DMY purity was as previously described (DMY, purity $>95 \%$, Fig. 1C).

\subsection{DMY attenuated the ethanol-induced hepatic enzyme release in mice}

The levels of the liver damage biomarkers ALT, AST, ALP and LDH were remarkably elevated by chronic alcohol ingestion. ( $\mathrm{P}<0.01$, Fig. $2 \mathrm{~A}, 2 \mathrm{~B}, 2 \mathrm{C}$ and $2 \mathrm{D}$ ). Nevertheless, DMY supplementation at doses of 75 and $150 \mathrm{mg} / \mathrm{kg}$ BW significantly reduced ALT, AST, ALP and LDH levels in serum compared with the alcohol control group $(\mathrm{P}<0.05$ or $\mathrm{P}<0.01$, Fig. $2 \mathrm{~A}, 2 \mathrm{~B}, 2 \mathrm{C}$ and 2D).

\subsection{DMY ameliorated lipid peroxidation and triglyceride accumulation in the liver}

The hepatic MDA level in liver tissue was significantly enhanced compared with that in the control group ( $\mathrm{P}<0.01$, Fig. $3 \mathrm{~A}$ ), whereas the GSH level was remarkably attenuated in the ethanol control group compared with that in the normal group $(\mathrm{P}<0.01$, Fig. $3 \mathrm{~B})$. However, the DMY groups (75 and $150 \mathrm{mg} / \mathrm{kg} \mathrm{BW}$ ) dramatically recovered these disordered changes in the liver due to the antioxidant activitiy of DMY ( $\mathrm{P}<0.01, \mathrm{P}<0.05$, Fig. $3 \mathrm{~A}$ and $3 \mathrm{~B})$. Subsequently, the frozen liver sections were stained with Oil Red $\mathrm{O}$, and the results revealed that hepatic lipid accumulation was obviously increased in chronic ethanol-stimulated mice in comparison with the control mice (Fig. 3C-a, b). In contrast, DMY supplementation significantly ameliorated the hepatic steatosis induced by chronic ethanol consumption in comparison with the alcohol-treated mice (Fig. 3C- b, c, d). 


\subsection{DMY alleviated IL-1 $\beta$ and IL-6 levels and pathological changes in the liver.}

IL-1 $\beta$ and IL- 6 are proinflammatory cytokines that play prominent roles in the pathogenesis of intrahepatic inflammation. We noticed that IL-1 $\beta$ and IL-6 levels were significantly elevated in livers compared to livers in control mice. ( $\mathrm{P}<0.01$, Fig. 4A, 4B). Nevertheless, both doses of DMY dramatically alleviated the hepatic IL-1 $\beta$ and IL-6 levels in comparison with those of the alcohol control mice $(\mathrm{P}<0.05$ or $\mathrm{P}<0.01$, Fig. $4 \mathrm{~A}$ and $4 \mathrm{~B})$. Afterwards, the $\mathrm{H} \& \mathrm{E}$ staining results showed that alcohol caused disordered hepatic changes, including obviously swollen hepatocytes, microvesicular steatosis, and inflammatory cell infiltration around the pericentral zone (Fig. 4C-a, b). The data were consistent with the alterations in hepatic lipid infiltration and inflammatory cytokine enhancement previously described. Nevertheless, administration with DMY at doses of 75 and $150 \mathrm{mg} / \mathrm{kg}$ BW remarkably ameliorated the liver histopathological changes induced by chronic ethanol exposure (Fig. 4C-b, c, d).

\subsection{DMY modified the aberrant expression of CYP2E1, Keap-1 and HO-1 in the liver.}

It was observed that the protein level of CYP2E1 was markedly enhanced, and simultaneously, the expression of HO-1 was significantly down-regulated in the ethanol-treated group compared with the control group ( $\mathrm{P}<0.01$, Fig. 5A and 5B). In contrast, the DMY groups showed a remarkably reversal of the aberrant expression of CYP2E1 and HO-1 in the liver compared to the ethanol group $(\mathrm{P}<0.05$ or $\mathrm{P}<0.01$, Fig. 5A and 5B). Intriguingly, the data suggested that ethanol exposure induced a slight attenuation of hepatic Keap-1 compared to control mice (Fig. 5A and 5C). Nevertheless, DMY administration significantly suppressed the expression of Keap-1 in the liver in comparison with the ethanol group $(\mathrm{P}<0.05$ or $\mathrm{P}<0.01$, Fig. 5A and 5C). Furthermore, we detected Keap1 mRNA levels using RT-PCR to further dissect the inhibitory effects of Keap-1 induced by DMY administration ( $\mathrm{P}>0.05$, Fig. 5D). Intriguingly, we noticed that there were no significant differences among all the groups, which indicated that DMY did not affect Keap1 mRNA levels. Hence, it is tempting to speculate that the DMY-induced down-regulation of the protein levels of Keap-1 was mediated by induction of Keap1 degradation. Consistently, immunohistochemistry results of Keap-1 in the livers visually supported the results from the western blot analyses and RT-PCR analyses. ( $\mathrm{P}<0.05$ or $\mathrm{P}<0.01$, Fig. $4 \mathrm{E}-\mathrm{a}, \mathrm{b}, \mathrm{c}$ and $\mathrm{d}$ ).

\subsection{DMY upregulated p62 and induced autophagy in the liver.}

Herein, in our study, we determined that chronic ethanol exposure significantly trigged the expression of p62 compared with control mice ( $\mathrm{P}<0.05$, Fig. 6A and 6B). Meanwhile, DMY administration dramatically enhanced p62 expression in comparison with the alcohol-treated mice 
( $\mathrm{P}<0.05$ or $\mathrm{P}<0.01$, Fig. $6 \mathrm{~A}$ and $6 \mathrm{~B})$. Furthermore, it was observed that ethanol significantly converted the soluble form of LC3-I to the lipidated LC3-II form and simultaneously increased the expression of Beclin 1 compared to control mice ( $\mathrm{P}<0.05$, Fig. 6A, 6C and 6D). In addition, DMY administration led to a further enhancement in the relative LC3-II level and the Beclin 1 expression, which are associated with autophagosomes, in comparison with the alcohol-treated mice $(\mathrm{P}<0.05$ or $\mathrm{P}<0.01$, Fig. $6 \mathrm{~A}, 6 \mathrm{C}$ and $6 \mathrm{D}$ ).

These results were confirmed by TEM, which has emerged as one of the most reliable measurements for autophagy, and multiple autophagolysosome-like vesicles were observed in hepatocytes. Notably, although proximal markers of autophagy significantly increased in ethanol-exposed mice, the TEM results revealed that ethanol did not increase the accumulation of autophagic vacuoles in the liver compared to control mice (Fig. 6E-a, b). Nevertheless, we noticed increased autophagolysosomes in the DMY groups compared to the ethanol-treated groups (Fig. $6 \mathrm{E}-\mathrm{c}, \mathrm{d})$.

\subsection{DMY ameliorated nuclear translocation of $N F-\kappa B$ and $N r f 2$ in the liver.}

The nuclear fractions were prepared, and the NF- $\mathrm{kB}$ and Nrf2 protein levels were determined using a western blot analysis. It appeared that the hepatic nuclear expression of Nrf2 was slightly down-regulated, whereas the nuclear expression of NF- $\mathrm{BB}$ was dramatically activated after chronic alcohol ingestion ( $\mathrm{P}<0.01$, Fig. 7A, 7B and 7C). Conversely, DMY administration significantly ameliorated the disordered expression of Nrf2 and NF- $\kappa B$ in hepatocyte nuclei compared with the ethanol treatment group $(\mathrm{P}<0.05$ or $\mathrm{P}<0.01$, Fig. 7A, 7B and $7 \mathrm{C})$.

Notably, the effect of DMY on the fluorescence localization of NF- $\mathrm{kB}$ and Nrf2 in ethanol-induced mice was in accord with the western blot results. The green, red and blue fluorescence represent the expression of Nrf2, NF- $\mathrm{KB}$ and DAPI, respectively. The immunofluorescence staining results confirmed that the green fluorescence intensity of Nrf2 in the nuclei was slightly attenuated, whereas the red fluorescence intensity of NF- $\mathrm{BB}$ in nuclei was remarkably enhanced due to long-term ethanol stimulation (Fig. 7D). In contrast, administration of DMY pronouncedly ameliorated the abnormal nuclear translocation of NF- $\mathrm{kB}$ and Nrf2 in the livers in comparison with that in livers from the ethanol group (Fig. 7D). Collectively, the present study visually consolidated the results indicating that DMY ameliorated nuclear translocation of $\mathrm{NF}-\mathrm{\kappa B}$ and Nrf2 in ethanol-induced mice.

\section{Discussion}

Chronic alcohol consumption is a predominant aetiological factor in the pathogenesis of ALD, and its clinical spectrum ranges from simple steatosis to fibrosis and hepatocellular carcinoma as previously described. According to a considerable number of literature reports, several mediators 
of ALD pathogenesis have been extensively identified and reviewed, including oxidative stress and inflammatory responses (Gao and Bataller, 2011; Miller et al., 2011). The development of effective agents for prevention of alcohol-induced hepatic steatosis and inflammation will depend on elucidation of the blockage of critical steps in ALD. Intriguingly, numerous preclinical and clinical trials have been performed with antioxidant agents, such as vitamins $\mathrm{C}$ and Silymarin, which ameliorate oxidative stress and consequently alleviate ethanol hepatotoxicity (Ramasamy and Agarwal, 2008; Abhilash et al., 2012).

Flavonoids are ubiquitously present in common plant-based functional compounds, which exert multi-targeted effects and have low toxicity, and flavonoids have recently attracted considerable attention as potential agents for ALD prevention (Ding et al., 2012). For instance, resveratrol and quercetin have been applied to suppress lipid synthesis and inflammation in the liver, which are closely associated with the activation of the Nrf2/HO-1 pathway (Kumar et al., 2011; Shi et al., 2013). Conspicuously, recent studies have creatively established that p62 can interact with Keap-1 and LC3B, leading to autophagic degradation of Keap-1, which contributes to a positive feedback loop of Nrf2 activation. The present study is the first to observe that the hepatoprotective effect of DMY is associated with modulation of the autophagy pathway crosstalk with the Keap-1-Nrf2 system through p62.

Chronic ethanol ingestion induces CYP2E1 activity enhancement and simultaneously leads to a reduction in proteasomal degradation and lipid peroxidation, which in turn dramatically boosts CYP2E1-induced aldehyde-protein adduct stability (Donohue et al., 2007; Osna and Donohue, 2007). Intriguingly, CYP2E1 deletion or administration of CYP2E1 inhibitors efficiently suppresses ethanol-mediated lipid peroxidation, whereas overexpression of CYP2E1 in transgenic mice can exacerbate hepatic injury compared to wild-type mice (Butura et al., 2009; Patouraux et al., 2012). In the current study, our results indicated that DMY down-regulated CYP2E1 expression to alleviate alcohol-induced hepatic steatosis and inflammation.

Nrf2 has recently been implicated as an essential transcription factor for alterations to phase I and II drug-metabolizing detoxification enzymes, and it protects against CYP2E1-dependent alcohol-induced liver injury (Zhou et al., 2014; Wu et al., 2012). Briefly, Nrf2 dissociates from Keap-1, translocates to the nucleus, binds to the antioxidant response element (ARE) and activates transcription aimed at cytoprotective and antioxidative factors, including HO-1 and GSH (Gong et al., 2014). In addition, available evidence in vivo and in vitro has demonstrated that blockage of Nrf2 aggravates mitochondrial damage and lipid accumulation in hepatocytes (Gong and Cederbaum, 2006; Lamlé et al., 2008). Moreover, activation of Nrf2 by hepatocyte-specific Keap-1 knockout in mice resulted in an enhancement in the NADPH concentration in the liver, which would be a protective target against oxidative stress (Wu et al., 2011). In addition, overwhelming evidence has indicated that oxidative stress can boost ROS generation and stimulate the NF- $\kappa B$ pathway while subsequently producing a massive secretion of proinflammatory cytokines, including IL-1 $\beta$ and IL-6 (Dhanda et al., 2012). In this study, our results showed that DMY 
remarkably attenuated the lipid peroxidation level and inflammatory response, followed by stimulation of Nrf2 nuclear translocation in the liver. Hence, the results of this study suggested that DMY protects against chronic ethanol-induced liver damage through activation of Nrf2. Nevertheless, the profound and underlying mechanisms of DMY modulation of the Nrf2 pathway should be further explored.

p62 (sequestosome 1, SQSTM1) is considered a multifaceted adaptor protein that possesses comprehensive biological features via interaction with numerous endogenous proteins (Moscat and Diaz-Meco, 2009). For instance, it has been revealed that p62 plays a crucial role in autophagy and serves as a molecular hub between autophagosomes and ubiquitin-conjugating protein aggregates in mammalian cells (Itakura and Mizushima, 2011). In particular, a recent work has reported that p62 also activated the Nrf2 pathway in a non-canonical manner. Substantial evidence has demonstrated that the formation of p62-Keap-1-LC3 protein complexes have been identified by mass spectrometry, immunoprecipitation (IP) and crystal structure analysis (Fan et al., 2010; Komatsu et al., 2010). Interestingly, genetic ablation of Keap1 resulted in the accumulation of ubiquitin aggregates, increased cytotoxicity of misfolded protein aggregates and defective activation of autophagy (Fan et al., 2010). Briefly, p62 activates Nrf2 through binding to Keap-1 at its KIR (residues 347-352), sequestering it, and subsequently interacts with LC3B, followed by autophagic degradation of Keap-1 and stabilization of Nrf2 (Ichimura et al., 2013). Intriguingly, accumulating evidence has demonstrated that Keap1 degradation is important for the maintenance of cellular redox homeostasis under oxidative stress (Taguchi et al., 2012). Notably, we noticed that DMY did not affect Keap1 mRNA levels, and therefore, it is tempting to speculate that down-regulation of the Keap-1 protein level by DMY was mediated by induction of Keap1 degradation. Collectively, the increased p62 expression induced by DMY can enhance the interaction of p62 with Keap-1, which is a substrate adaptor of the Cul3-ubiquitin ligase complex, leading to Nrf2 degradation (Kobayashi et al., 2004). More importantly, the upregulated p62 protein can also interact with LC3B, and p62 upregulation led to autophagic degradation of Keap-1, which was responsible for a positive feedback loop of Nrf2 activation. Indeed, this finding is consistent with the results of the western blot analyses and immunohistochemistry examination of Keap-1 and Nrf2 previously described.

Intriguingly, several studies have indicated that accumulation of p62 in addition to ubiquitylated protein aggregates results in various disorders, which are caused by disruption of Nrf2 degradation and its persistent activation under autophagy-deficient conditions (Duleh et al., 2016). Notably, to date, the biological functions of p62 under diverse pathophysiological processes remain elusive and modulating functions of accumulated p62 in liver disease have seldom been reported in autophagy sufficiency conditions. Intriguingly, several studies have indicated that upregulation of Nrf2 activity in a p62-dependent manner plays a protective role to alleviate oxidative injury in the liver (Bae et al., 2013). Consistently, recent evidence established that 
ezetimibe could activate the p62-mediated Keap-1/Nrf2 pathway without causing cytotoxicity (Lee et al., 2016). Recently, autophagy, which maintains cellular functions through lysosomal degradation of damaged proteins, has been recognized as a potential therapeutic target for liver diseases (Puri and Chandra, 2014). LC3B is used as a marker to monitor autophagy and, especially lipidated LC3-II, is linked to autophagosome formation and induction of autophagy (Hu et al., 2012). In addition, Beclin1 has emerged as a pivotal modifier in the autophagic process due to its ability to cooperate with the phosphatidylinositol-3 (PI3K) kinase pathway to increase the formation of autophagic vacuoles (García-Mauriño et al., 2012). In the current study, we noticed that DMY administration enhanced the relative LC3-II level and Beclin 1 expression, which is associated with autophagosomes. Simultaneously, ethanol was also able to enhance the expression of proximal markers of autophagy, whereas the TEM results revealed that ethanol did not increase the accumulation of autophagic vacuoles in hepatocytes. Regarding the effect of ethanol on autophagy, recent studies have determined that chronic ethanol-triggered impairment of autophagic flux in the liver contributes to attenuation of autophagosome-lysosome fusion (Cho et al., 2014). Nevertheless, DMY significantly elevated the excessive accumulation of autophagolysosomes, which eventually progressed to autophagosome fusion with lysosomes and subsequently led to cellular content degradation. Indeed, we observed that DMY dramatically induced autophagy, and the results are supported by numerous reports (Xia et al., 2014; Shi et al., 2015). In particular, these findings explain that DMY significantly stimulated p62 expression and subsequently contributed to activation of Nrf2 under conditions of autophagy sufficiency. Briefly, DMY-induced autophagy probably provides beneficial and sufficient autophagic conditions to confirm p62-mediated Keap-1 degradation. Collectively, DMY upregulation of p62 and the autophagy pathway facilitated Keap-1 degradation, which in turn contributed to a positive feedback loop in Nrf2 activation.

\section{Conclusion}

Our present study demonstrated that DMY serves as an antioxidant flavonoid that likely activates the Nrf2/Keap-1 pathway to protect against liver injury induced by chronic alcoholic consumption. Mechanistically, in this study, we investigated the crosstalk among p62, Keap-1, and Nrf2 and further observed that p62 upregulation induced by DMY contributes to a positive feedback loop in the Keap-1/Nrf2 pathway. Notably, DMY dramatically induced autophagy and possibly provides beneficial and sufficient autophagic conditions to confirm p62-mediated Keap-1 degradation. Taken together, on the basis of these results, our studies provide novel mechanistic insights into the hepatoprotective effect of DMY, which may be exploited as a therapeutic option for liver injury induced by chronic ethanol consumption. 


\section{Acknowledgements}

This study was supported by grants from the Project of the State of Traditional Chinese Medicine Industry special project (No. 201507002), the Science of Chinese Traditional Medicine in the first academic subject of Zhejiang Province top priority, and the National Science Foundation of China (Grant No. 81303224). 


\section{References}

Abhilash, P.A., Harikrishnan, R., Indira, M., 2012. Ascorbic acid supplementation down-regulates the alcohol induced oxidative stress, hepatic stellate cell activation, cytotoxicity and mRNA levels of selected fibrotic genes in guinea pigs. Free Radical Res. 46, 204-213.

Bae, S.H., Sung, S.H., Oh, S.Y., Lim, J.M., Lee, S.K., Park, Y.N., Lee, H.E., Kang, D., Rhee, S.G., 2013. Sestrins activate Nrf2 by promoting p62-dependent autophagic degradation of Keap1 and prevent oxidative liver damage. Cell Metab. 17, 73-84.

Bardag-Gorce, F., Oliva, J., Lin, A., Li, J., French, B.A., French, S.W., 2011. Proteasome inhibitor up regulates liver antioxidative enzymes in rat model of alcoholic liver disease. Exp. Mol. Pathol. 90, 123-130.

Bataille, A.M., Manautou, J.E., 2012. Nrf2: a potential target for new therapeutics in liver disease. Clin. Pharmacol. Ther. 92, 340-348.

Butura, A., Nilsson, K., Morgan, K., Morgan, T.R., French, S.W., Johansson, I., Ingelman-Sundberg, M., 2009. The impact of CYP2E1 on the development of alcoholic liver disease as studied in a transgenic mouse model. J. Hepatol. 50, 572-583.

Ceni, E., Mello, T., Galli, A., 2014. Pathogenesis of alcoholic liver disease: role of oxidative metabolism. World J. Gastroenterol. 20, 17756-17772.

Cho, H.I., Choi, J.W., Lee, S.M., 2014. Impairment of autophagosome-lysosome fusion contributes to chronic ethanol-induced liver injury. Alcohol. 48, 717-25.

Ding, R.B., Tian, K., He, C.W., Jiang, Y., Wang, Y.T., Wan, J.B., 2012. Herbal medicines for the prevention of alcoholic liver disease: a review. J. Ethnopharmacol. 144, 457-465.

Dhanda, A.D., Lee, R.W., Collins, P.L., McCune, C.A., 2012. Molecular targets in the treatment of alcoholic hepatitis. World J. Gastroenterol. 18, 5504-5513.

Donohue, T.M., Cederbaum, A.I., French, S.W., Barve, S., Gao, B., Osna, N.A., 2007. Role of the Proteasome in Ethanol-Induced Liver Pathology. Alcohol. Clin. Exp. Res. 31, 1446-1459.

Duleh, S., Wang ,X., Komirenko, A., Margeta, M., 2016. Activation of the Keap1/Nrf2 stress response pathway in autophagic vacuolar myopathies. Acta Neuropathol. Commun. 4, 115.

Fan, W., Tang, Z., Chen, D., Moughon, D., Ding, X., Chen, S., Zhu, M., Zhong, Q., 2010. Keap1 facilitates p62-mediated ubiquitin aggregate clearance via autophagy. Autophagy 6, 614-21.

Gao, B., Bataller, R., 2011. Alcoholic liver disease: pathogenesis and new therapeutic targets. Gastroenterology 141, 1572-1585.

García-Mauriño, S., Alcaide, A., Domínguez, C., 2012. Pharmacological control of autophagy: therapeutic perspectives in inflammatory bowel disease and colorectal cancer. Curr. Pharm. Des. 26, 3853-73.

Gong, P., Cederbaum, A.I., 2006. Nrf2 is increased by CYP2E1 in rodent liver and HepG2 cells and protects against oxidative stress caused by CYP2E1. Hepatology 43, 144-153. 
Gong, P., Stewart, D., Hu, B., Li, N., Cook, J., Nel, A., Alam, J., 2002. Activation of the mouse heme oxygenase-1 gene by 15 -deoxy- $\Delta 12,14$-prostaglandin $\mathrm{J} 2$ is mediated by the stress response elements and transcription factor Nrf2. Antioxid. Redox Sign. 4, 249-257.

Gyamfi, M.A. and Wan, Y.J.Y., 2010. Pathogenesis of alcoholic liver disease: the role of nuclear receptors. Exp. Biol. Med. 235, 547-560.

Hayashi, K., Dan, K., Goto, F., Tshuchihashi, N., Nomura, Y., Fujioka, M., Kanzaki, S., Ogawa, K., 2015. The autophagy pathway maintained signaling crosstalk with the Keap1-Nrf2 system through p62 in auditory cells under oxidative stress. Cell Signal. 27, 382-93.

Hu, C., Zou, M.J., Zhao, L., Lu, N., Sun, Y.J., Gou, S.H., Xi, T., Guo, Q.L., 2012. E Platinum, a newly synthesized platinum compound, induces autophagy via inhibiting phosphorylation of mTOR in gastric carcinoma BGC-823 cells. Toxicol. Lett. 210, 78-86.

Ichimura, Y., Waguri, S., Sou, Y.S., Kageyama, S., Hasegawa, J., Ishimura, R., Saito, T., Yang, Y., Kouno, T., Fukutomi, T., Hoshii, T., Hirao, A., Takagi, K., Mizushima, T., Motohashi, H., Lee, M.S., Yoshimori, T., Tanaka, K., Yamamoto, M., Komatsu, M., 2013. Phosphorylation of p62 activates the Keap1-Nrf2 pathway during selective autophagy, Mol. Cell. 51, 618-631.

Itakura, E., Mizushima, N., 2011. p62 Targeting to the autophagosome formation site requires self-oligomerization but not LC3 binding. J. Cell Biol. 192, 17-27.

Jain, A., Lamark, T., Sjøttem, E., Larsen, K.B., Awuh, J.A., Øvervatn, A., McMahon, M., Hayes, J.D., Johansen, T., 2010. p62/SQSTM1 is a target gene for transcription factor NRF2 and creates a positive feedback loop by inducing antioxidant response element-driven gene transcription. J. Biol. Chem. 285, 22576-91.

Jiang, B., Le, L., Pan, H., Hu, K., Xu, L., Xiao, P., 2014. Dihydromyricetin ameliorates the oxidative stress response induced by methylglyoxal via the AMPK/GLUT4 signaling pathway in PC12 cells. Brain Res. Bull. 109, 117-126.

Kobayashi, A., Kang, M.I., Okawa, H., Ohtsuji, M., Zenke, Y., Chiba, T., Igarashi, K., Yamamoto, M., 2004. Oxidative stress sensor Keap1 functions as an adaptor for Cul3-based E3 ligase to regulate proteasomal degradation of Nrf2. Mol. Cell Biol. 24, 7130-9.

Kobayashi, M., Yamamoto, M., 2005. Molecular mechanisms activating the Nrf2-Keap1 pathway of antioxidant gene regulation. Antioxid. Redox Sign. 7, 385-394.

Komatsu, M., Kurokawa, H., Waguri, S., Taguchi, K., Kobayashi, A., Ichimura, Y., Sou, Y.S., Ueno, I., Sakamoto, A., Tong, K.I., Kim, M., Nishito, Y., Iemura, S., Natsume, T., Ueno, T., Kominami, E., Motohashi, H., Tanaka, K., Yamamoto, M., 2010. The selective autophagy substrate p62 activates the stress responsive transcription factor Nrf2 through inactivation of Keap1. Nat. Cell Biol. 12, 213-23.

Kumar, A., Singh, C.K., LaVoie, H.A., DiPette, D.J., Singh, U.S., 2011. Resveratrol restores Nrf2 level and prevents ethanol-induced toxic effects in the cerebellum of a rodent model of fetal alcohol spectrum disorders. Mol. Pharmacol. 80, 446-457. 
Kumar, K.S., Liao, J.W., Xiao, J.H., Vani, M.G., Wang, S.Y., 2012. Hepatoprotective effect of lucidone against alcohol-induced oxidative stress in human hepatic HepG2 cells through the up-regulation of HO-1/Nrf-2 antioxidant genes. Toxicol. in Vitro 26, 700-708.

Lamlé, J., Marhenke, S., Borlak, J., Von Wasielewski, R., Eriksson, C.P., Geffers, R., Vogel, A., 2008. Nuclear Factor-Eythroid 2-Related Factor 2 Prevents Alcohol-Induced Fulminant Liver Injury. Gastroenterology 134, 1159-1168.

Lee, D.H., Han, D.H., Nam, K.T., Park, J.S., Kim, S.H., Lee, M., Kim, G., Min, B.S., Cha, B.S., Lee, Y.S., Sung, S.H., Jeong, H., Ji, H.W., Lee, M.J., Lee, J.S., Lee, H.Y., Chun, Y., Kim, J., Komatsu, M., Lee, Y.H., Bae, S.H., 2016. Ezetimibe, an NPC1L1 inhibitor, is a potent Nrf2 activator that protects mice from diet-induced nonalcoholic steatohepatitis. Free Radic. Biol. Med. 99, 520-532.

Li, S., Tan, H.Y., Wang, N., Zhang, Z.J., Lao, L.X., Wong, C.W., Feng, Y.B., 2015. The Role of Oxidative Stress and Antioxidants in Liver Diseases. Int. J. Mol. Sci. 16, 26087-26124.

Miller, A.M., Horiguchi, N., Jeong, W.I., Radaeva, S., Gao, B., 2011. Molecular mechanisms of alcoholic liver disease: innate immunity and cytokines. Alcohol. Clin. Exp. Res. 35, 787-793.

Moscat, J., Diaz-Meco, MT., 2009. p62 at the crossroads of autophagy, apoptosis, and cancer. Cell 137, 1001-1004.

Nepal, S., Park, P. H., 2013. Activation of autophagy by globular adiponectin attenuates ethanol-induced apoptosis in HepG2 cells: involvement of AMPK/FoxO3A axis. Biochim. Biophy. Acta 1833, 2111-2125.

Orman, E.S., Odena, G., Bataller, R., 2013. Alcoholic liver disease: pathogenesis, management, and novel targets for therapy. J. Gastroien. Hepatol. 28, 77-84.

Osna, N.A., Donohue, T.M., 2007. Implication of altered proteasome function in alcoholic liver injury. World J. Gastroenterol. 13, 4931.

Park, J.S., Kang, D.H., Lee, D.H., Bae, S.H., 2015. Fenofibrate activates Nrf2 through p62-dependent Keap1 degradation. Biochem. Biophys. Res. Commun. 465, 542-7.

Patouraux, S., Bonnafous, S., Voican, C.S., Anty, R., Saint-Paul, M.C., Rosenthal-Allieri, M.A., Agostini, H., Njike, M., Barri-Ova, N., Naveau, S., Le Marchand-Brustel, Y., 2012. The osteopontin level in liver, adipose tissue and serum is correlated with fibrosis in patients with alcoholic liver disease. PloS One 7, e35612.

Puri, P., Chandra, A., 2014. Autophagy modulation as a potential therapeutic target for liver diseases. J. Clin. Exp. Hepatol. 4, 51-9.

Ramasamy, K., Agarwal, R., 2008. Multitargeted therapy of cancer by silymarin. Cancer Lett. 269, 352-362.

Shen, Y., Lindemeyer, A.K., Gonzalez, C., Shao, X.M., Spigelman, I., Olsen, R.W., Liang, J., 2012. Dihydromyricetin as a novel anti-alcohol intoxication medication. J. Neurosci. 32, 390-401. 
Shi, L., Zhang, T., Liang, X., Hu, Q., Huang, J., Zhou, Y., Chen, M., Zhang, Q., Zhu, J., Mi, M., 2015. Dihydromyricetin improves skeletal muscle insulin resistance by inducing autophagy via the AMPK signaling pathway. Mol. Cell Endocrinol. 409, 92-102.

Shi, Y., Liang, X.C., Zhang, H., Wu, Q.L., Qu, L., Sun, Q., 2013. Quercetin protects rat dorsal root ganglion neurons against high glucose-induced injury in vitro through $\mathrm{Nrf-2/HO}-1$ activation and NF-кB inhibition. Acta Pharmacol. Sin. 34, 1140-1148.

Taguchi, K., Fujikawa, N., Komatsu, M., Ishii, T., Unno, M., Akaike, T., Motohashi, H., Yamamoto, M., 2012. Keap1 degradation by autophagy for the maintenance of redox homeostasis. P. Natl. Acad. Sci. USA. 109, 13561-6.

Teli, M.R., Day, C.P., James, O.F. W., Burt, A.D., Bennett, M.K., 1995. Determinants of progression to cirrhosis or fibrosis in pure alcoholic fatty liver. Lancet 346, 987-990.

Wang, W., Guan, C., Sun, X., Zhao, Z., Li, J., Fu, X., Yuwen Q., Min H., Jin, J., Huang, Z., 2016. Tanshinone IIA protects against acetaminophen-induced hepatotoxicity via activating the Nrf2 pathway. Phytomedicine 23, 589-596.

Wu, K.C., Cui, J.Y., Klaassen, C.D., 2011. Beneficial role of Nrf2 in regulating NADPH generation and consumption. Toxicol. Sci. 123, 590-600.

Wu, K.C., Liu, J., Klaassen, C.D., 2012. Role of Nrf2 in preventing ethanol-induced oxidative stress and lipid accumulation. Toxicol. App. Pharmacol. 262, 321-329.

Xia, J., Guo, S., Fang, T., Feng, D., Zhang, X., Zhang, Q., Liu, J., Liu, B., Li, M., Zhu, R., 2014. Dihydromyricetin induces autophagy in HepG2 cells involved in inhibition of mTOR and regulating its upstream pathways. Food Chem. Toxicol. 66, 7-13.

Zhou, R., Lin, J., Wu, D., 2014. Sulforaphane induces Nrf2 and protects against CYP2E1-dependent binge alcohol-induced liver steatosis. Biochim. Biophys. Acta 1840, 209-218. 


\section{Figure captions}

Fig. 1. The chemical structre and chromatographic analysis of DMY. (A): The power of DMY (B): The chemical structre of DMY. (C): The DMY content was analysed by HPLC in this study and the purity of DMY was determined to be $>95 \%$; the black arrow indicates the dihydromyricetin.

Fig. 2. The effects of DMY administration on hepatic enzyme release in mice fed a Lieber-DeCarli liquid diet for 6 weeks (n=12). (A, B, C, D): DMY administration significantly inhibited the activities of ALT, AST, ALP and LDH in serum in chronic ethanol-exposed mice. Significant differences are indicated by $* \mathrm{P}<0.05$ and $* * \mathrm{P}<0.01$.

Fig. 3. Effects of DMY administration on the levels of MDA, GSH and lipid deposition in ethanol-treated mice. (A, B): DMY supplementation significantly inhibited the augmentation of the hepatic MDA level and simultaneously enhanced the hepatic GSH activity. (C): Oil Red O staining shows the excessive cytoplasmic lipid accumulation dramatically attenuated by DMY administration $(\times 200)$. Significant differences are indicated by $* \mathrm{P}<0.05$ and $* * \mathrm{P}<0.01$.

Fig. 4. The effects of DMY administration on the hepatic inflammatory cytokine release and pathomorphology changes in mice with chronic ethanol exposure. $(n=12)(A, B)$ : The effects of DMY on IL-1 $\beta$ and IL-6 levels in livers were quantified with ELISAs. (C): H\&E (haematoxylin and eosin) staining confirmed that DMY remarkably alleviated ethanol-induced lipid deposition and inflammatory cell infiltration $(\times 400)$. Significant differences are indicated by $* \mathrm{P}<0.05$ and $* *$ $\mathrm{P}<0.01$.

Fig. 5. Effects of DMY administration on hepatic CYP2E1, Keap-1 and HO-1 expression in ethanol-induced mice. (A): CYP2E1, Keap-1 and HO-1 protein levels in the liver were detected with western blotting analyses and quantified with Tubulin as an internal control. (B, C): CYP2E1, Keap-1 and HO-1 protein levels are expressed as the relative content of the control value. These data are representative of three independent experiments. (D): Keap-1 mRNA expression level in the liver was detected with RT-PCR analyses and normalized to GAPDH. These data are representative of three independent experiments. (E): Immunohistochemistry results of Keap1 in the livers confirmed that DMY significantly inhibited the expression of Keap-1 in ethanol-treated mice. a: Normal, b: EtOH, c: DMY $(75 \mathrm{mg} / \mathrm{kg})$, d: DMY $(75 \mathrm{mg} / \mathrm{kg})$. Significant differences are indicated by $* \mathrm{P}<0.05$ and $* * \mathrm{P}<0.01$.

Fig. 6. Administration of DMY upregulated p62 and induced autophagy in ethanol-induced mice. (A): p62, LC3B and Beclin 1 protein levels were quantified, with $\beta$-actin as an internal control. (B, C, D) DMY remarkably upregulated the expression of p62 and Beclin 1 and LC3 lipidation in a 
dose-dependent manner. These data are representative of three independent experiments. (E): Autophagic vacuoles (indicated by red arrows) were observed in TEM micrographs and indicated that DMY dramatically induced autophagy in ethanol-treated mice. a: Normal, b: EtOH, c: DMY $(75 \mathrm{mg} / \mathrm{kg})$, d: DMY $(75 \mathrm{mg} / \mathrm{kg})$. Significant differences are indicated by $* \mathrm{P}<0.05$ and $* *$ $\mathrm{P}<0.01$.

Fig. 7. The effects of DMY on the nuclear expression and fluorescence localization of Nrf2 and $\mathrm{NF}-\mathrm{\kappa B}$ in livers with chronic ethanol exposure. (A) NF- $\mathrm{KB}$ and Nrf2 protein levels were quantified using lamin B1 as a nucleoprotein internal control. (B, C) NF- $\kappa \mathrm{B}$ and Nrf2 protein levels are expressed as the relative content of the control value. (D) The green, red and blue fluorescence represent the expression of Nrf2, NF- $\mathrm{KB}$ and DAPI, respectively. The merged image represents the colocalization of Nrf2, NF-kB and DAPI. DMY dramatically stimulated nuclear translocation of

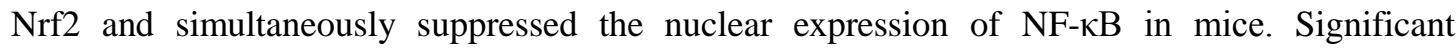
differences are indicated by $* \mathrm{P}<0.05$ and $* * \mathrm{P}<0.01$. 
A



Dihydromyricetin

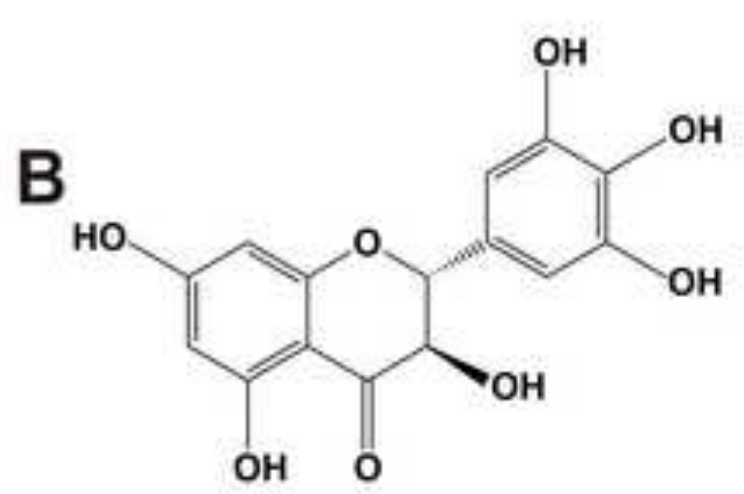

C

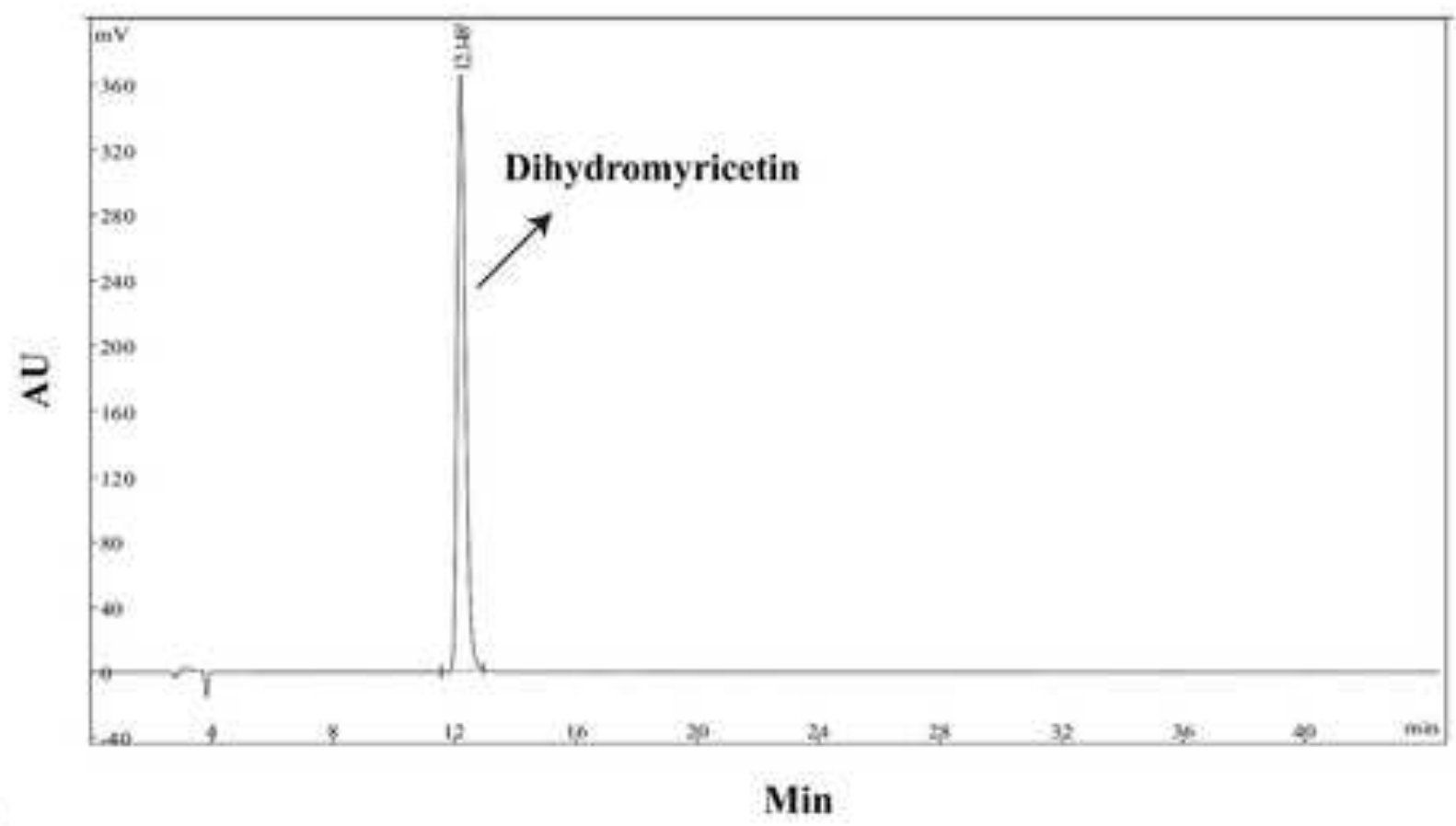

chemical structure of Dihydromyricetin 


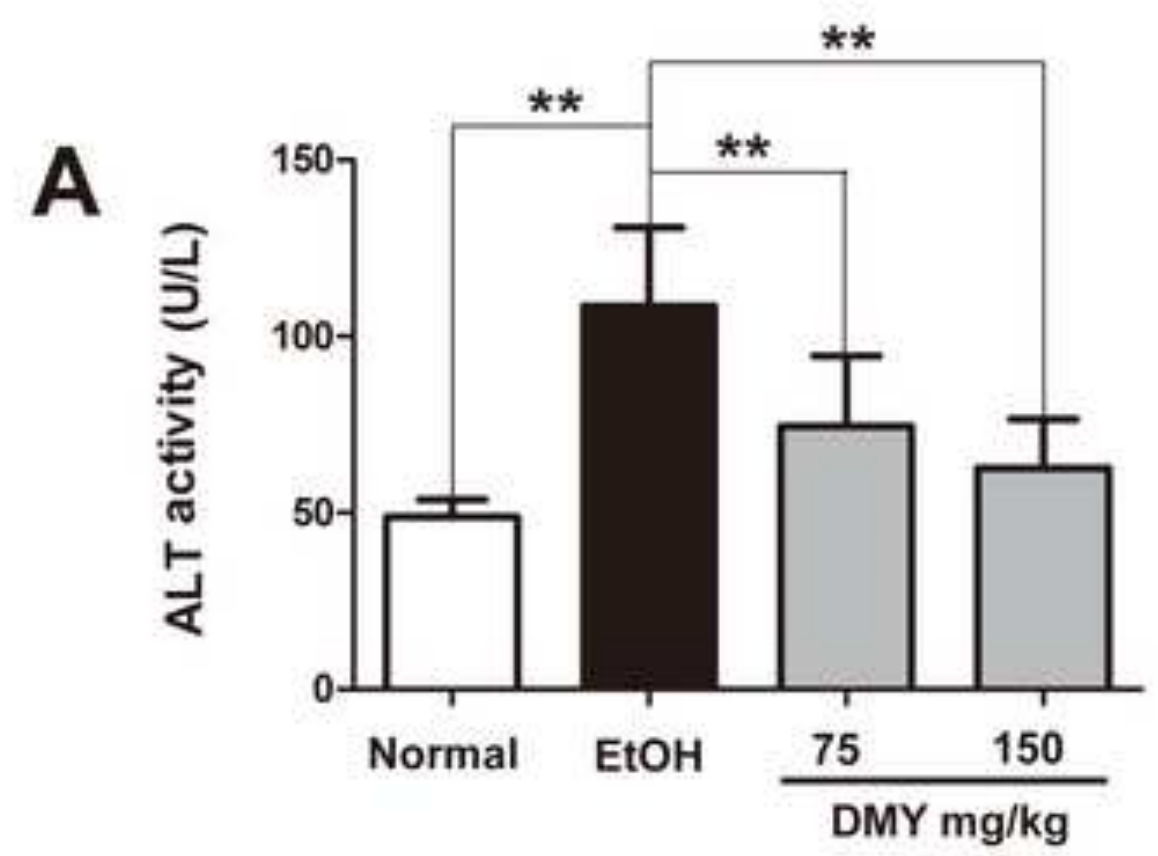

B

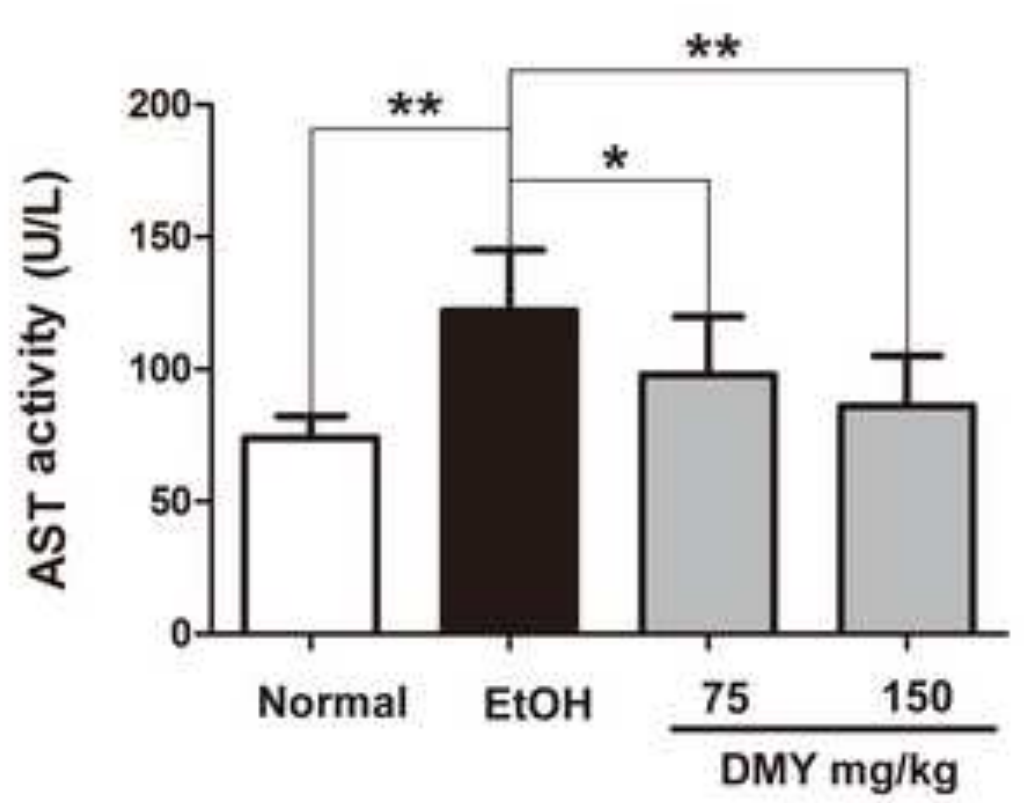

C
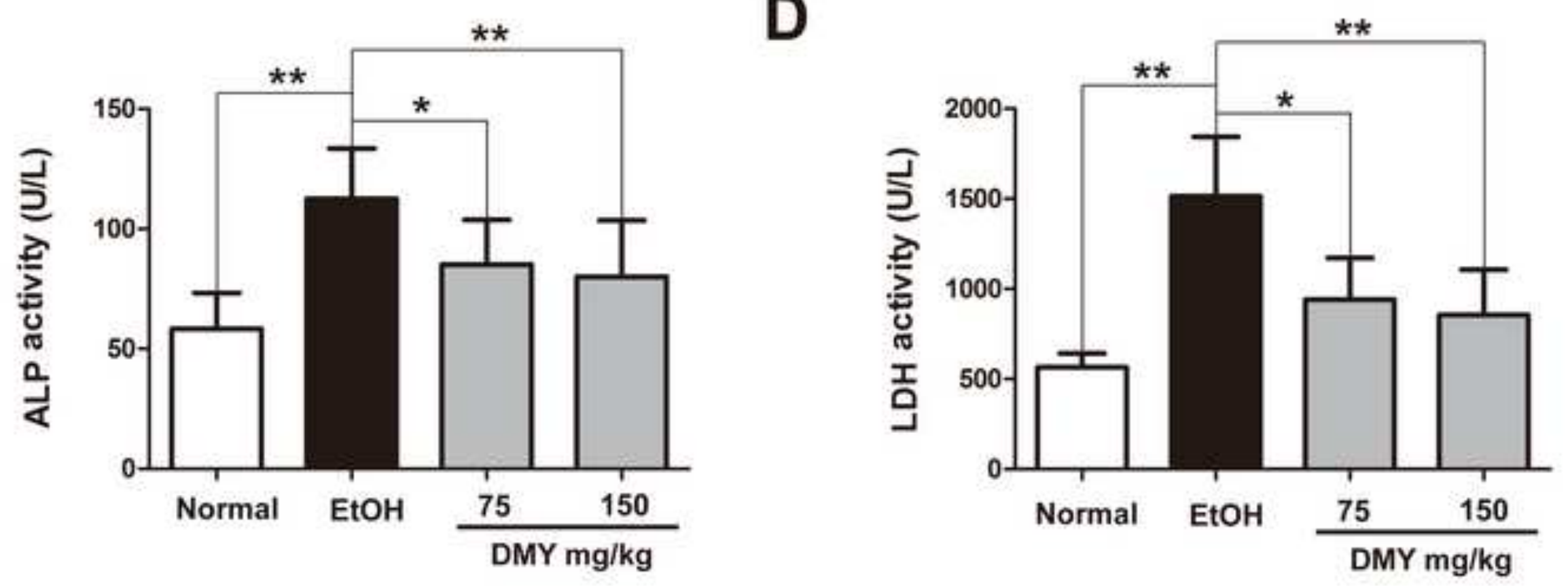
A

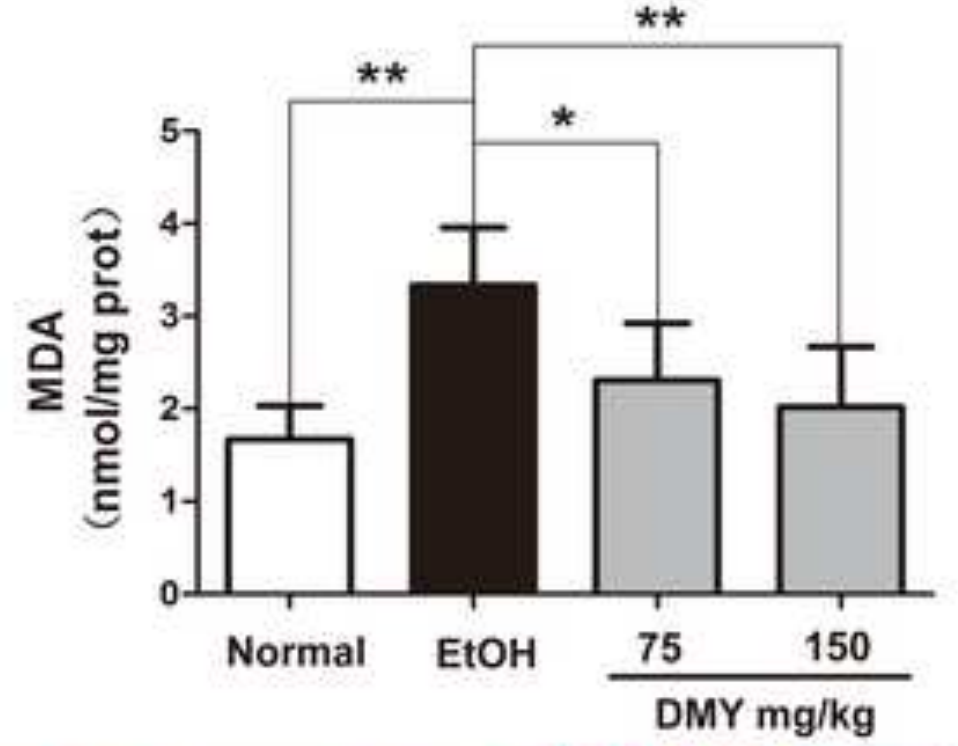

C

Oil Red O



Normal
B



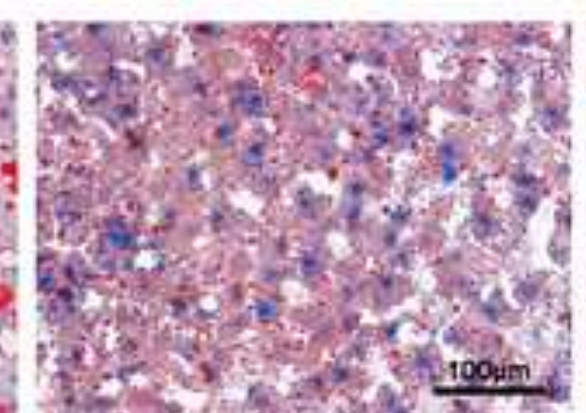

DMY $(75 \mathrm{mg} / \mathrm{kg})$

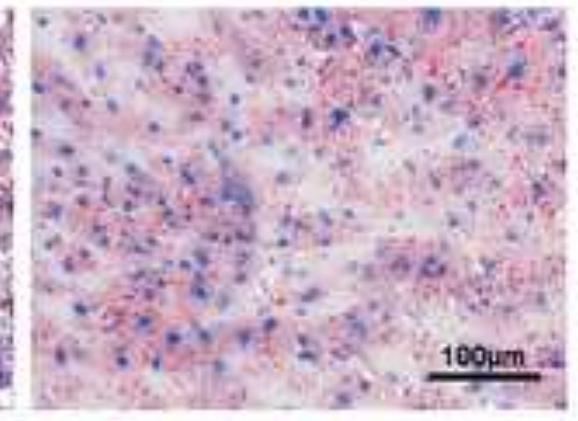

DMY $(150 \mathrm{mg} / \mathrm{kg})$ 
A

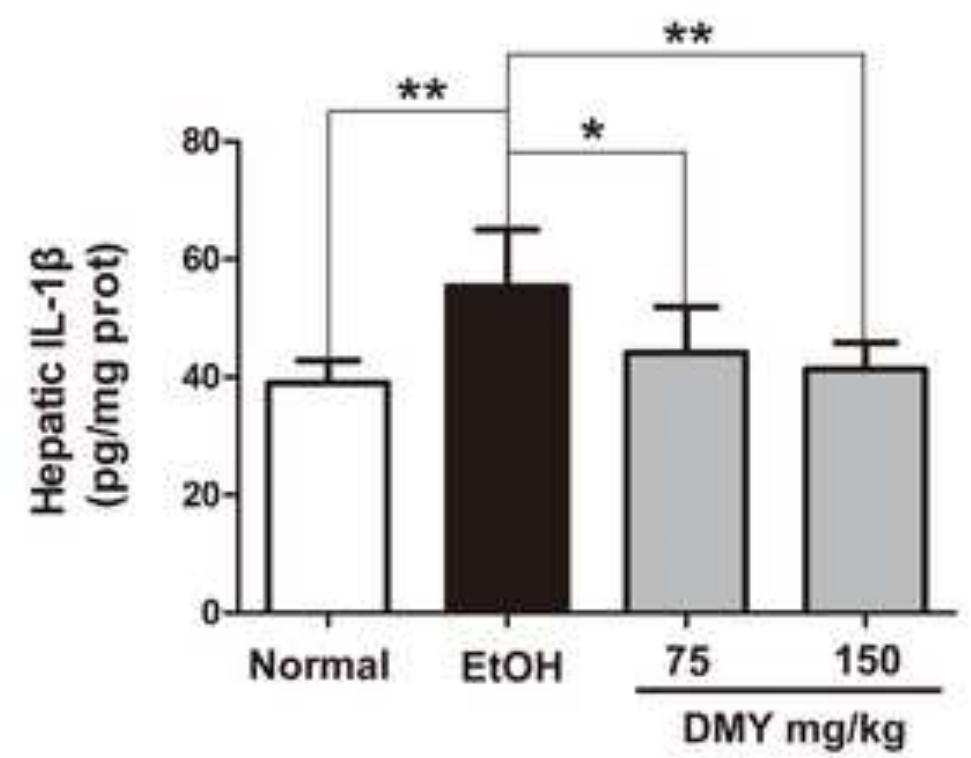

C

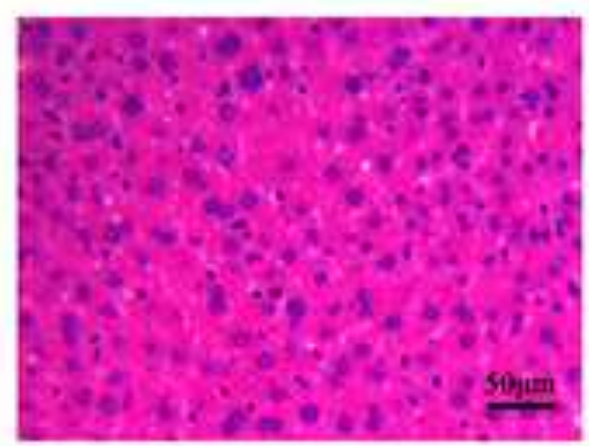

Normal



EtOH
B

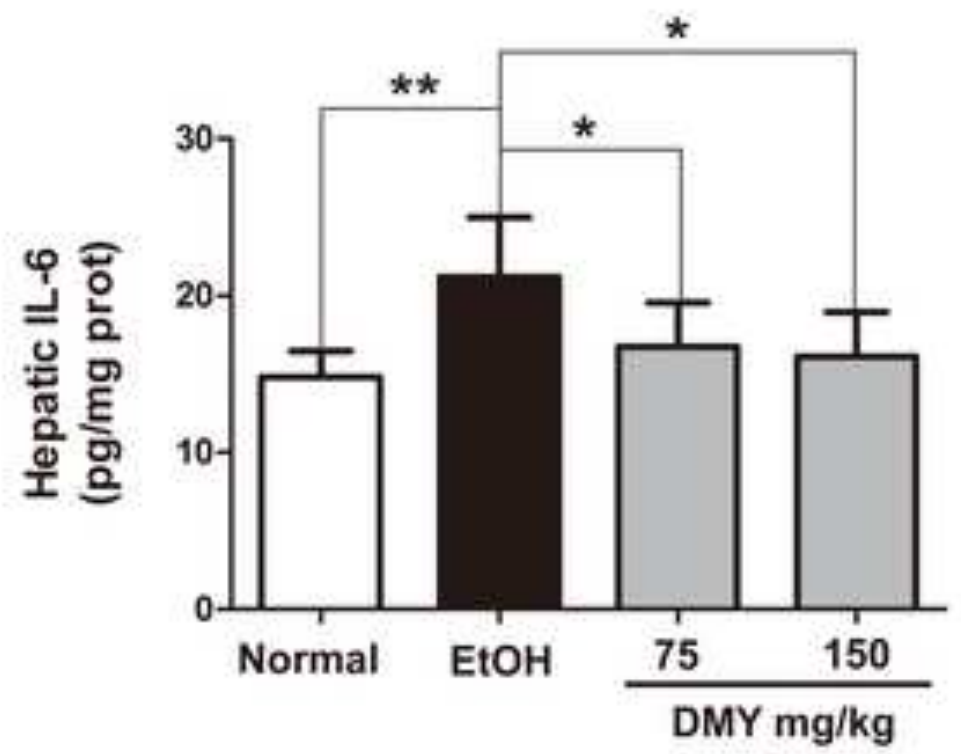



DMY $(75 \mathrm{mg} / \mathrm{kg})$

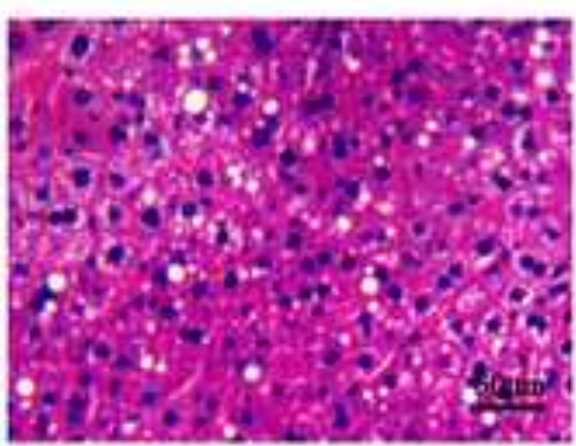

DMY $(150 \mathrm{mg} / \mathrm{kg})$ 
A

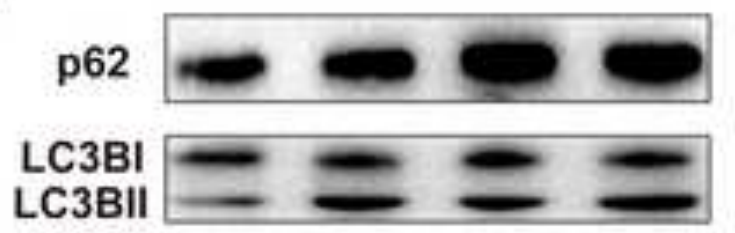

Beclin 1
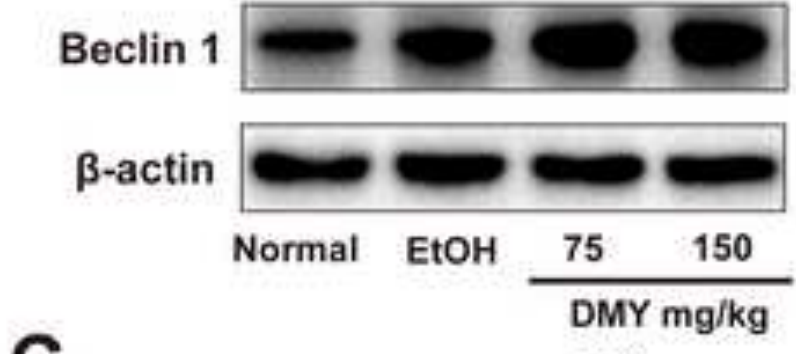

C
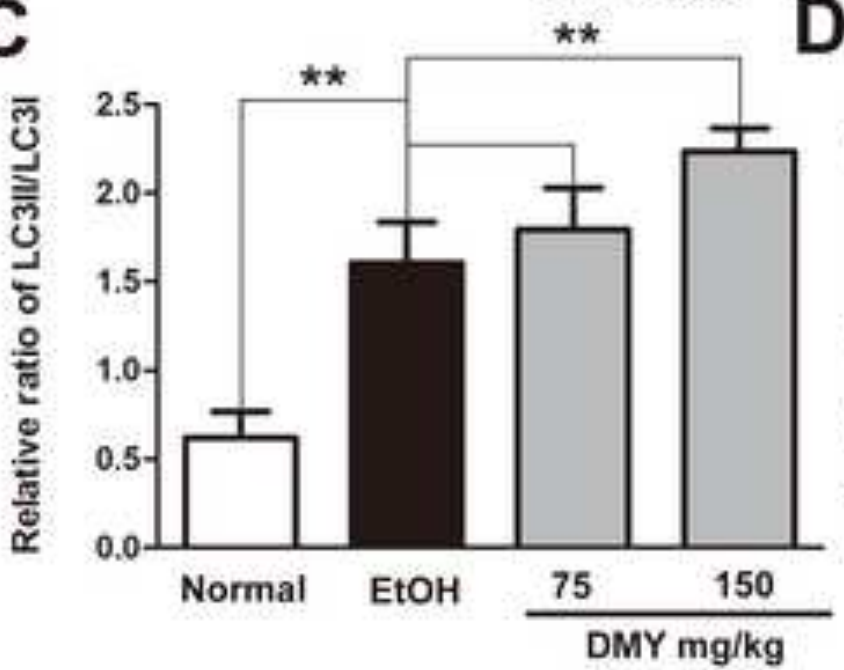

E
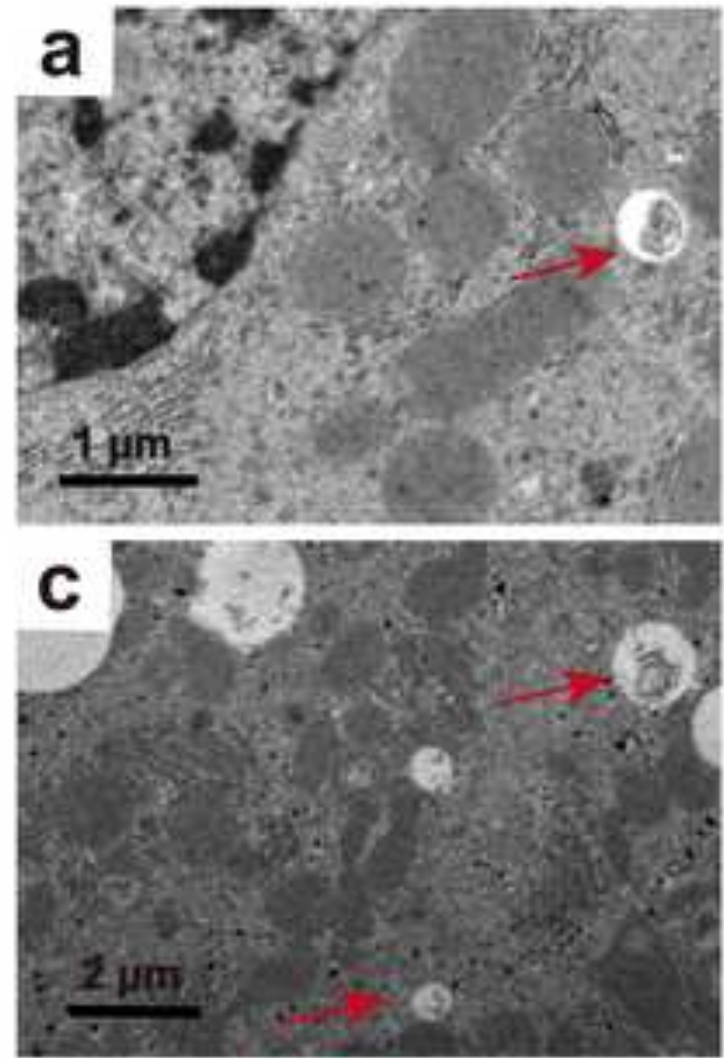

B
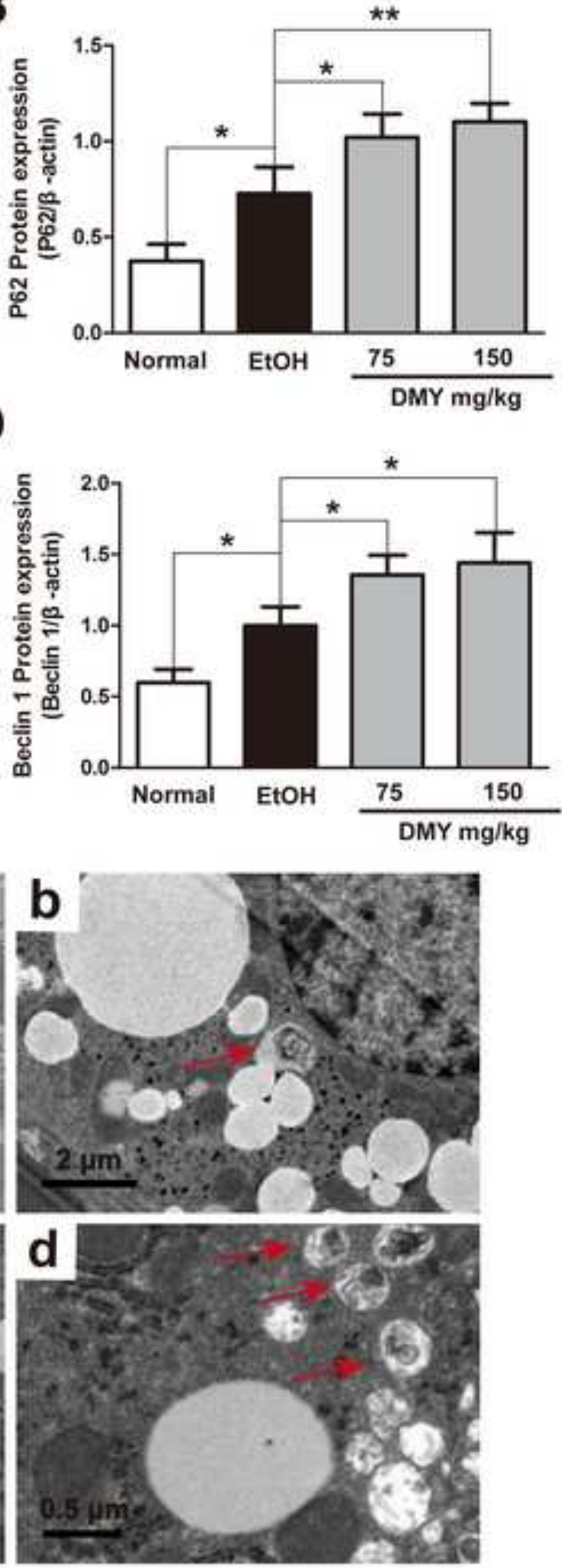

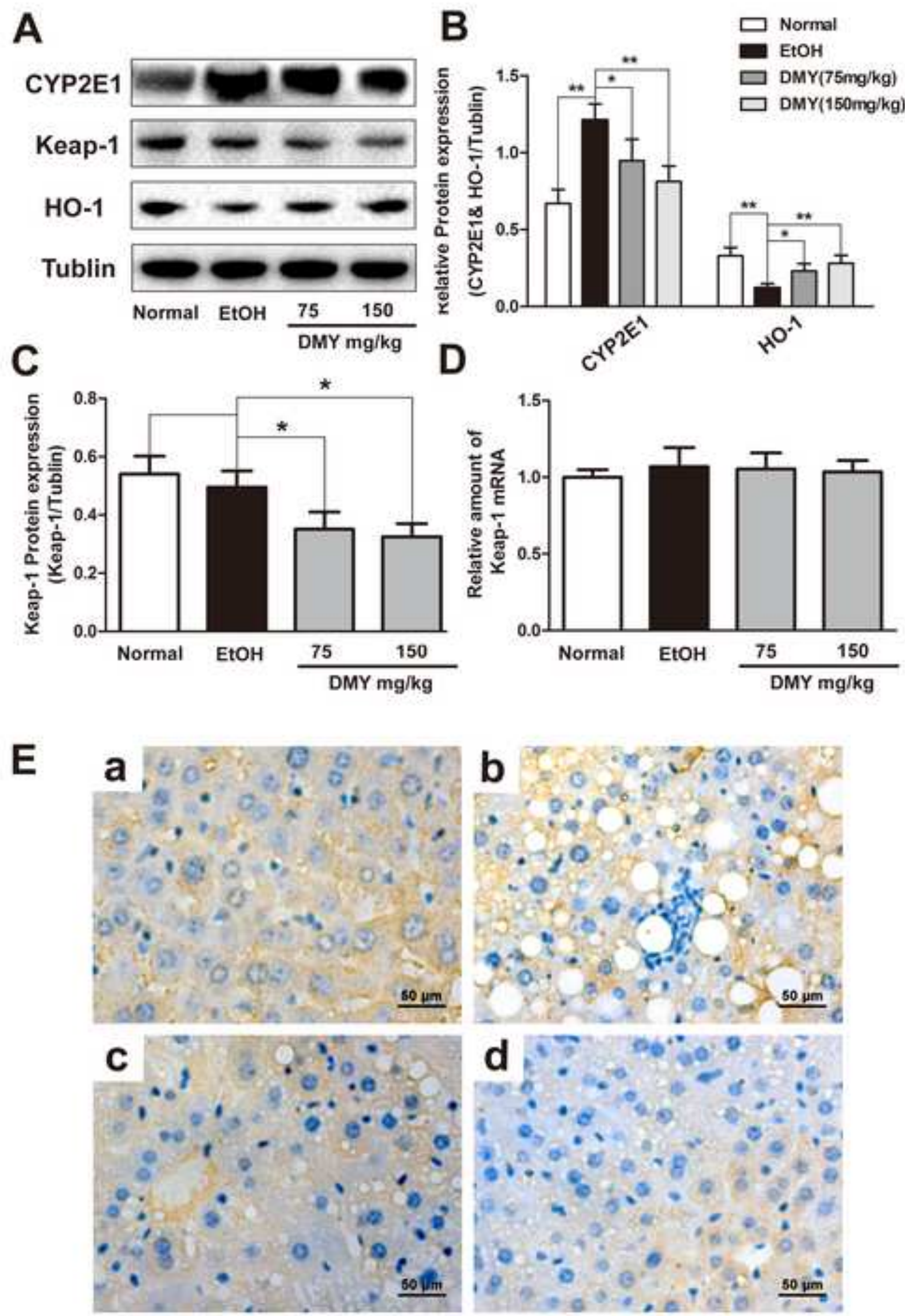


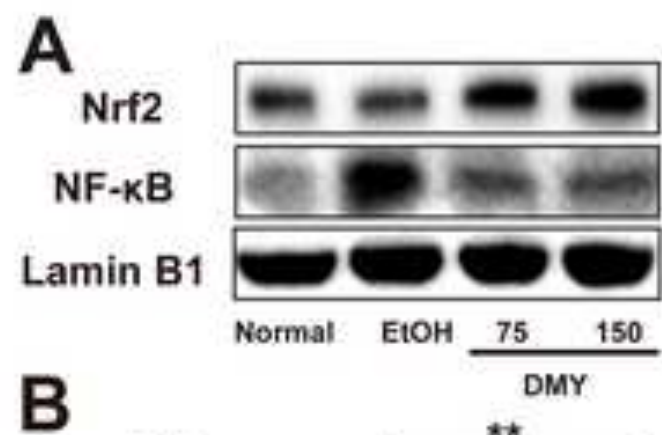

D

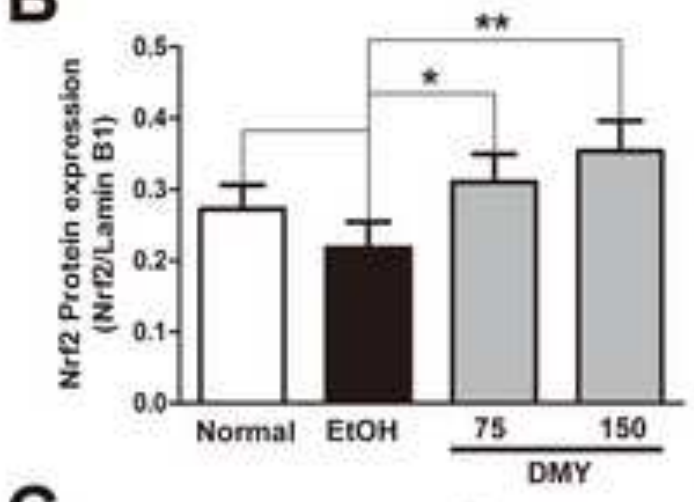

C

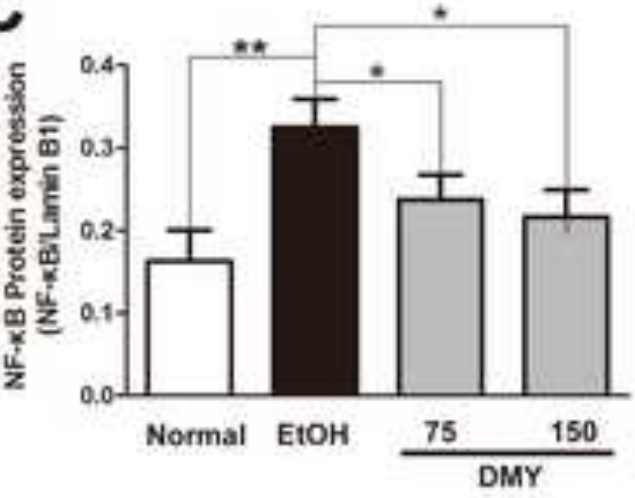

$\mathrm{Nr} 2$
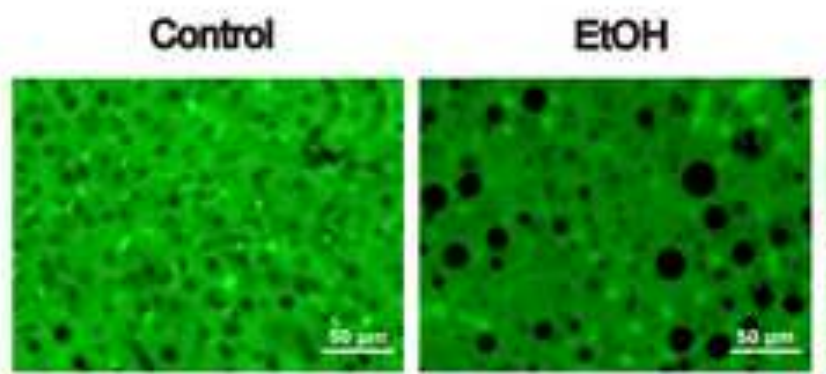

DMY $(75 \mathrm{mg} / \mathrm{kg})$

DMY(150mg/kg)
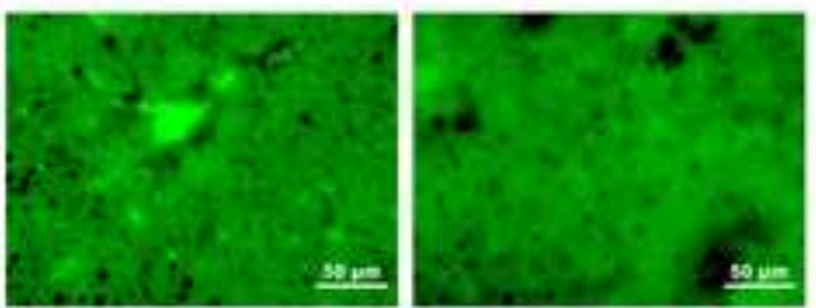

$\mathrm{NF}-\mathrm{KB}$
DAPI
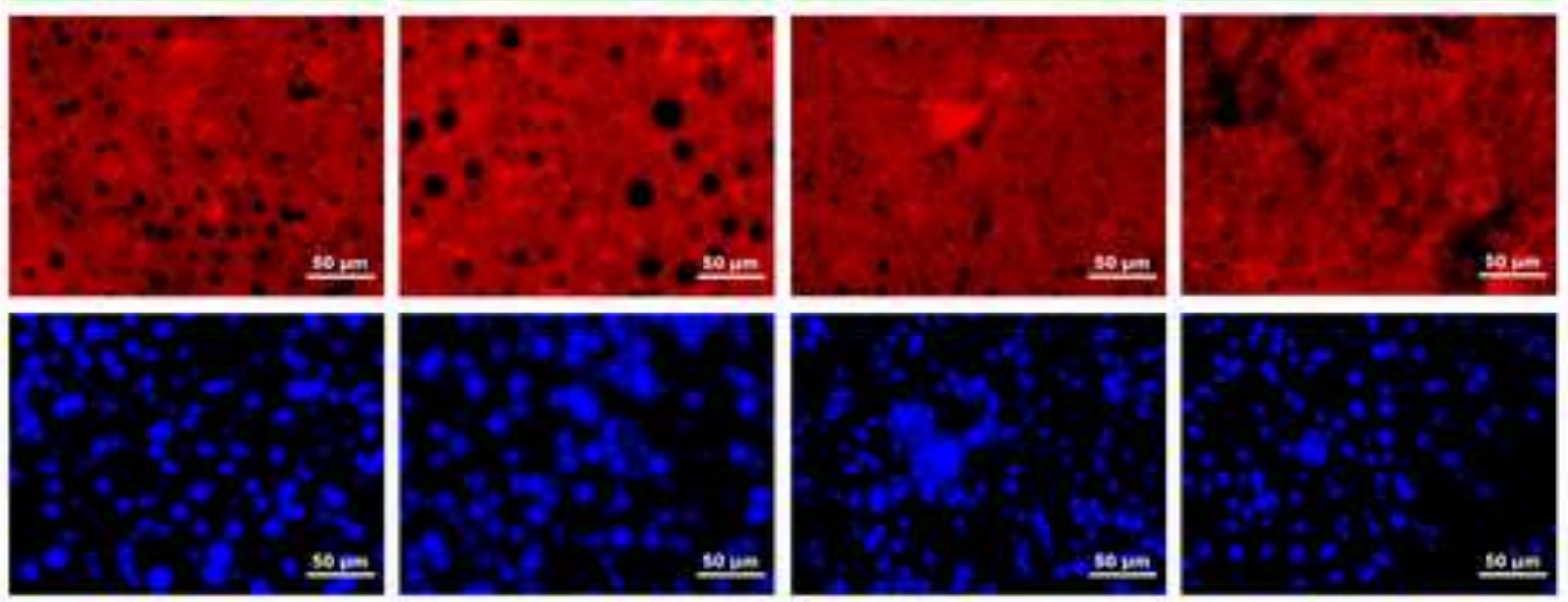

Merge


19un 